\title{
Suplementação com probióticos e aminoácidos no desempenho de novilhos de corte: estudo de identidade de modelos
}

\author{
Supplementation with probiotics and amino acids in the development of beef cattle: \\ identity model study
}

\section{OLIVEIRA, Marcus Vinícius Moraes de ${ }^{1}$; SILVA, Ana Paula Viscardi da²; BONATTI, Franscine Kelli Quinhones ${ }^{1 *}$; MARCATTI, Gustavo Eduardo ${ }^{3}$; VARGAS JUNIOR, Fernando Miranda de ${ }^{4}$}

\footnotetext{
${ }^{1}$ Universidade Estadual de Mato Grosso do Sul, Departamento de Zootecnia, Aquidauana, Mato Grosso do Sul, Brasil.

${ }^{2}$ Universidade Federal de Mato Grosso do Sul, Faculdade de Medicina Veterinária e Zootecnia, Campo Grande, Mato Grosso do Sul, Brasil.

${ }^{3}$ Universidade Federal de Viçosa, Departamento de Engenharia Florestal, Viçosa, Minas Gerais, Brasil.

${ }^{4}$ Universidade Federal da Grande Dourados, Faculdade de Ciências Agrária, Dourados, Mato Grosso do Sul, Brasil.

*Endereço para correspondência: franbonatti@gmail.com
}

\section{RESUMO}

O objetivo deste estudo foi avaliar o ganho de peso de novilhos suplementados com probióticos e aminoácidos, por meio da análise de identidade de modelos. Foram utilizados 64 novilhos castrados da raça Canchim e cruzados Canchim x Nelore com $24 \pm 1,8$ meses de idade e peso corpóreo inicial de 395,4 $\pm 10,5 \mathrm{~kg}$, mantidos em pastagem de Brachiaria brizantha em sistema de pastejo contínuo. Foram testados quatro tratamentos: Testemunha (Test); Probiótico líquido (ProbL); Probiótico líquido mais aminoácidos em pó (ProbL+AAsPó); e Probiótico líquido mais aminoácido injetável (ProbL+AAsInj), em um delineamento experimental inteiramente casualizado com 16 animais por tratamento. Os tratamentos foram comparados através do Teste de Identidade de Modelos, utilizando o software R. O modelo logístico de três parâmetros (L3p) foi o selecionado para representar o ganho de peso médio diário em função do tempo. As estimativas dos parâmetros do modelo foram significativas em todos os tratamentos, apresentando coeficiente de determinação $\left(\mathrm{R}^{2}\right)$ elevado e raiz quadrada do erro quadrático médio (RMSE) baixa. De acordo com o teste de identidade de modelos não houve diferença significativa entre os modelos para os tratamentos utilizados, não havendo diferença no ganho de peso dos animais com a utilização de probióticos e/ou de aminoácidos.

Palavras-chave: aditivo, bovinos, ganho de peso, teste de verossimilhança

\section{SUMMARY}

The goal of this study was to analyze the weight gain of calves supplemented with probiotics and amino acids, through model identity analysis. There were utilized 64 catrated Canchin and Canchin x Nelore calves at $24 \pm 1,8$ months of age and initial average weight of $395,4 \pm 10,5$ $\mathrm{Kg}$. The calves were kept on continuous grazing system in a Brachiária brizantha pasture. Four treatments were evaluated: witnesses (test); probiotic liquid (ProbL); probiotic liquid plus amino acids powder (ProbL+AAsPó); and probiotic liquid plus injectable amino acids, in a randomized experimental design with 16 animals by each treatment. The treatments, in turn, were compared trough identity model test, on $\mathrm{R}$ software. In order to represent the daily average weight gain over time, the logistic model with three parameters (L3p) was selected. The parameter's estimates of the model were significant in every treatment, with high 
coefficient of determination $\left(\mathrm{R}^{2}\right)$ and low root mean square error (RMSE). There was not significant difference between the models for the treatments, based on the identity model test. Therefore, there was no difference in weight gain by probiotics and/or amino acids utilization.

Keywords: additive, cattle, weight gain, likelihood test

\section{INTRODUÇÃO}

O Brasil é o maior exportador mundial de carne bovina e um dos poucos países, entre os exportadores, que possui potencial de aumentar ainda mais sua produção em quantidade e qualidade. Para isso, novas tecnologias vêm sendo testadas com intuito de aumentar a produção de carne em menor tempo, principalmente em animais criados a pasto. Neste sentido, os probióticos e aminoácidos naturais, apresentam-se como uma das alternativas, pois potencializam e melhoram a eficiência alimentar, aumentando o crescimento e o ganho de peso dos animais.

Os probióticos são utilizados com o intuito de melhorar o equilíbrio da flora intestinal, harmonizando a função digestiva e a saúde do animal (VANBELLE et al., 1990). O aumento no desempenho animal com a utilização desses produtos, segundo Morais et al. (2011), está relacionado a produção de compostos antimicrobianos, competição com microrganismos indesejáveis pela colonização do substrato, produção de nutrientes, produção ou estímulo de enzimas, metabolismo ou detoxificação de compostos indesejáveis e estímulo de resposta imune. Por outro lado, a utilização de suplementos com funções orgânicas, compostos basicamente por aminoácidos naturais, vitaminas e minerais (SILVA et al., 2002), visam a estimulação da produção hormonal das glândulas hipófise, tireóide e paratireóide (BALDWIN et al., 1994) promovendo a reconstituição protéica e retenção de nitrogênio, com reflexos positivos no ganho de peso (CORRÊA et al.,1998; CAMPOS NETO et al., 2003).

A comercialização de probióticos e aminoácidos vêm crescendo nos últimos anos, principalmente no período de menor precipitação, onde a disponibilidade de pasto é menor. Todavia, há uma falta de dados comprobatórios quanto à eficácia desses produtos, não sendo comumente encontrados dados científicos na literatura. Ressalta-se também que a avaliação de dados pelo método de identidade de modelos não é uma prática muito utilizada em estudos conduzidos com bovinos, no entanto, pode ser aplicada em estudos de desenvolvimento de animais, como afirma Carneiro et al. (2014), com o objetivo de verificar a possibilidade de ajuste de equações comuns para diferentes grupos de indivíduos, e para testar hipóteses com relação ao modelo e aos seus respectivos parâmetros.

Neste sentido, o objetivo deste trabalho foi avaliar o efeito de probióticos $\mathrm{e}$ aminoácidos naturais sobre o ganho de peso de novilhos corte por meio da análise de testes de identidade de modelos.

\section{MATERIAL E MÉTODOS}

O experimento foi realizado na Fazenda Escola Três Barras, no município de Campo Grande - MS, no período de outono-inverno, entre os meses de março a julho. Foram utilizados 64 novilhos castrados, da raça Canchim e cruzados Canchim x Nelore, com $24 \pm 1,8$ meses de idade e peso corpóreo inicial de 395,4 $\pm 10,5 \mathrm{~kg}$. 
Rev. Bras. Saúde Prod. Anim., Salvador, v.17, n.3, p.474-483 jul./set., $2016 \quad \underline{\text { http://www.rbspa.ufba.br }}$ ISSN 15199940

Os animais foram distribuídos em 16 piquetes de sete hectares cada. Em cada piquete foi alocado um grupo de quatro animais, compondo a unidade experimental do experimento. Foram testados quatro tratamentos em um delineamento experimental inteiramente casualizado com quatro repetições, contendo 16 animais. A variável utilizada para representar o desempenho foi o ganho de peso médio diário. Os animais foram pesados no inicio do experimento e posteriormente a cada 28 dias, após 12 horas de jejum de líquidos e sólidos, totalizando 112 dias de período experimental, e cinco pesagens.

Os tratamentos utilizados foram: Testemunha (Test); Probiótico líquido (ProbL); Probiótico líquido mais aminoácidos em pó (ProbL+AAsPó); e Probiótico líquido mais aminoácidos injetável (ProbL+AAsInj). A utilização dos probióticos e aminoácidos seguiu a orientação do fabricante, assim, o ProbL foi na dose de $5 \mathrm{~mL} /$ animal no inicio do experimento e depois $1 \mathrm{~mL} / \mathrm{animal} /$ dia até o fim do experimento, o AAsPó na dose de 5g/animal no inicio do experimento e depois $1 \mathrm{~g} /$ animal/dia até o fim do experimento, e o AAsInj na dose de $10 \mathrm{~mL} /$ animal em três aplicações, sendo a primeira no inicio do experimento, e as outras duas em intervalos de 28 dias. O ProbL e o AAsPó foram misturados ao concentrado e então fornecido aos animais. Já o AAsInj, foi aplicado via intramuscular.

A composição dos probióticos e dos aminoácidos são apresentadas nas Tabelas 1, 2 e 3. A pastagem utilizada foi a Brachiaria brizantha cv. Marandu, implantada a cinco anos em solo caracterizado como Latossolo Vermelho Distroférrico (EMBRAPA, 2001) corrigido e adubado na época da implantação. O sistema de pastejo foi contínuo com carga animal fixa.
Tabela 1. Composição do Probiótico Líquido (ProbL)

\begin{tabular}{lcc}
\hline Ingredientes & Níveis de garantia \\
\hline $\begin{array}{l}\text { Bifidobacterium } \\
\text { (mínimo) }\end{array}$ & bifidum & $3,33 \times 10^{6} \mathrm{ufc} / \mathrm{g}$ \\
$\begin{array}{l}\text { Enterococcus } \\
\text { (mínimo) }\end{array}$ & faecium & $1,66 \times 10^{6} \mathrm{ufc} / \mathrm{g}$ \\
$\begin{array}{l}\text { Lactobacillus acidophilus } \\
\text { Lactobacillus plantarum }\end{array}$ & $3,33 \times 10^{6} \mathrm{ufc} / \mathrm{g}$ \\
$\begin{array}{l}\text { Saccharomyces cerevisiae } \\
\text { ufc/g = unidades formadoras de colônias por }\end{array}$ & $3,33 \times 10^{6} \mathrm{ufc} / \mathrm{g}$ \\
\hline $\begin{array}{l}\text { grama. } \\
l\end{array}$
\end{tabular}

Tabela 2. Composição do Aminoácido em Pó (AAsPó)

\begin{tabular}{lc}
\hline Ingredientes & Níveis de garantia \\
\hline Ácido Glutâmico & $14,0 \mathrm{mg}$ \\
L-lisina & $33,0 \mathrm{mg}$ \\
Metionina & $7,0 \mathrm{mg}$ \\
Histidina & $7,0 \mathrm{mg}$ \\
Triptofano & $2,0 \mathrm{mg}$ \\
\hline
\end{tabular}

Tabela 3. Composição do Aminoácido Líquido (AAsInj)

\begin{tabular}{lr}
\hline Ingredientes & $\begin{array}{r}\text { Níveis de } \\
\text { garantia }\end{array}$ \\
\hline Hidrolisados de órgão e & $5.000,0 \mathrm{mg}$ \\
glândulas & $420,0 \mathrm{mg}$ \\
L-Acido Glutâmico & $1.000,0 \mathrm{mg}$ \\
L- Lisina Cloridrato & $210,0 \mathrm{mg}$ \\
Acetil Metionina & $60,0 \mathrm{mg}$ \\
L- Triptofano & $210,0 \mathrm{mg}$ \\
L- Histidina Cloridrato & $16,0 \mathrm{mg}$ \\
Sódio & $3,0 \mathrm{mg}$ \\
Magnésio & $42,0 \mathrm{mg}$ \\
Cobre & $15,0 \mathrm{mg}$ \\
Manganês & $8,0 \mathrm{mg}$ \\
Zinco & $10,0 \mathrm{mg}$ \\
Ferro Dextrano & $500,0 \mathrm{mg}$ \\
Vitamina B1 (Cloridrato de & $500,0 \mathrm{mg}$ \\
Tiamina) & $200,0 \mathrm{mg}$ \\
Vitamina B2 (Cloridrato de & $100,0 \mathrm{ml}$ \\
Piridoxina) & \\
Niacina (Ácido Nicotínico) & Água Destilada q.s.p.
\end{tabular}


Todos os animais receberam $600 \mathrm{~g}$ de concentrado contendo $95 \%$ de milho triturado e $5 \%$ de melaço em pó. Também foi fornecido sal mineral e núcleo proteico, contendo $40 \%$ de proteína bruta, com consumo diário estimado de $60 \mathrm{~g}$ e $500 \mathrm{~g}$ por animal, respectivamente.

Os tratamentos foram comparados através do teste de identidade de modelos (REGAZZI \& SILVA, 2010). Para a aplicação deste teste foram realizados os seguintes procedimentos: (1) utilização de medidas de qualidade de ajuste, para determinar qual é o modelo mais adequado para representar o ganho de peso dos animais em função do tempo. Para isso foi calculado o coeficiente de determinação $\left(\mathrm{R}^{2}\right)$, raiz quadrada do erro quadrático médio
(RMSE) e dispersão dos resíduos; (2) após a escolha do(s) modelo(s), segundo os critérios citados acima, os modelos foram ajustados para cada um dos tratamentos; e (3) aplicação do teste de razão de verossimilhança, a $5 \%$ de significância, entre cada modelo(s) ajustado(s) aos tratamentos para averiguar a identidade dos modelos, e se houve diferenças entre os tratamentos.

Foram avaliados 13 modelos: Intercepto (Int); Linear Simples (LS); Linear Quadrático (LQ); Exponencial Modificado (EM); Michaelis Menten (MM); Associação Exponencial 2 e 3 parâmetros (AE2) (AE3); Assintótico (ASS); Logistico 3 e 4 parâmetros (L3p) (L4p); Gompertz (Gom); Exponencial 2 e 3 parâmetros (Exp2) (Exp3); os quais são apresentados na Tabela 4.

Tabela 4. Modelos avaliados

\begin{tabular}{llc}
\hline Sigla & & Modelo \\
\hline 1 & Int & $y=\beta_{1}+e$ \\
2 & LS & $\beta_{1} * x+\beta_{2}+e$ \\
3 & LQ & $y=\beta_{1} * x^{2}+\beta_{2} * x+\beta_{3}+e$ \\
\hline 4 & EM & $y=\beta_{1} * \exp \left(\beta_{2} / x\right)+e$ \\
5 & MM & $y=\beta_{1} * x /\left(\beta_{2}+x\right)+e$ \\
\hline 6 & AE3 & $y=\beta_{1} *\left(b-\exp \left(-\beta_{2} * x\right)+e\right.$ \\
7 & AE2 & $y=\beta_{1} *\left(1-\exp \left(-\beta_{2} * x\right)+e\right.$ \\
8 & Ass & $y=\left(\beta_{2}-\beta_{1}\right) * \exp \left(-\exp \left(\beta_{3}\right) * x\right)+e$ \\
9 & L3p & $y=\beta_{1} /\left(1+\exp \left(\left(\beta_{2}-x\right) / \beta_{3}\right)\right)+e$ \\
10 & L4p & $\beta_{1}+\left(\beta_{2}-\beta_{1}\right) /\left(1+e x p\left(\left(\beta_{3}-x\right) / \beta_{4}\right)\right)+e$ \\
11 & Gom & $y=\beta_{1} * \exp \left(-\beta_{2} * \beta_{3}{ }^{x}\right)+e$ \\
\hline 12 & Exp2 & $y=\beta_{1} * \exp \left(x * \beta_{2}\right)+e$ \\
13 & Exp3 & $y=\beta_{1}+\beta_{2} * \exp \left(x / \beta_{3}\right)+e$ \\
\hline Int-Intercepto; LS- Linear Simples; LQ- Linear Quadrático; EM- Exponencial Modificado; MM- & Miç \\
Michaelis Menten; AE2- Associação Exponencial 2 parâmetros; AE3- Associação Exponencial 3 \\
parâmetros; ASS- Assintótico; L3p- Logístico 3 parâmetros; L4p- Logístico 4 parâmetros; Gom- \\
Gompertz; Exp2- Exponencial 2 parâmetros; Exp3- Exponencial 3 parâmetros.
\end{tabular}


Os modelos foram ajustados via mínimos quadrados generalizados não lineares com a utilização da função gnls da biblioteca nlme do software R. As demais análises também foram realizadas no software $\mathrm{R}$.

\section{RESULTADOS E DISCUSSÃO}

Entre os modelos testados para representar o ganho de peso dos animais em função do tempo, o modelo Logístico 3 parâmetros (L3p) (Eq. 1) foi o selecionado, pois apresentou os melhores resultados com relação às medidas de qualidade de ajuste. As estimativas dos parâmetros do modelo foram significativas em todos os tratamentos (Tabela 5), apresentando $\mathrm{R}^{2}$ elevado e RMSE baixa. Por meio destas duas medidas, juntamente com a avaliação do gráfico de dispersão dos resíduos, foi possível constatar que o modelo Lp3 foi o que melhor se ajustou aos dados, e consequentemente o que apresentou maior capacidade de representar os tratamentos aplicados neste estudo. Este modelo é da classe dos modelos não lineares, com crescimento sigmoide, cuja curva tem início num ponto fixo aumentando à taxa de crescimento monotônicamente até chegar a um ponto de inflexão, depois disso, a taxa de crescimento se aproxima de um valor final assintoticamente. Neste estudo, ele foi capaz de representar as medidas de ganho de peso dos animais distribuídas ao longo do tempo, uma vez que, esta distribuição se assemelha a curvas sigmoides. Segundo Carneiro et al. (2014), as curvas de crescimento e ganho de peso de bovinos, podem ser descritas por modelos que consideram a relação não linear entre o peso e a idade dos animais. Os modelos não lineares são os mais indicados para o estudo biológico de diferentes espécies (RATKOWSKY, 1990), pois representa de forma adequada o processo de crescimento, com menor número de parâmetros do que modelos lineares, além de apresentarem interpretação biológica (SOUZA et al., 2010; SILVA et al., 2011) o que facilita o estudo do ganho de peso do animal ao longo do tempo.

$$
y=\beta_{1} /\left(1+\exp \left(\left(\beta_{2}-x\right) / \beta_{3}\right)\right)+e \quad(\text { Eq. } 1)
$$

Em que: $\gamma=$ ganho de peso; $x=$ tempo (dias); $\beta_{1}=$ assíntota, quando $x \rightarrow+\infty$, ou seja, o máximo valor teórico de $y(x)$; $\beta_{2}=$ tempo de "meia-vida", ou seja, o valor de $x$ correspondente $\beta_{1} / 2 ; \beta_{3}=$ taxa de crescimento médio de $y(x)$.

$\mathrm{Na}$ representação gráfica do modelo nos tratamentos utilizados (Figura 1), o valor máximo (Max) de ganho de peso aos 112 dias foi semelhante entre os tratamentos e variou de 57,21 a $62,66 \mathrm{Kg}$. O ganho de peso diário e o peso corpóreo final dos animais (Tabela 6) também foram semelhantes entre os tratamentos. O ganho de peso médio dos animais neste estudo pode ser considerado baixo quando comparado ao ganho de peso de novilhos Nelores em pastagens de Brachiaria brizantha com a utilização de bioestimulantes a base de aminoácidos observados por Castro et al. (2009). Da mesma maneira que o ganho de peso relatado por Figueiredo et al. (2012) avaliando a suplementação com resíduo úmido de cervejaria, associado a utilização de aminoácidos, em novilhos da raça Nelore também em pastagem de Brachiaria brizantha. No entanto, Soutello et al. (2002) encontraram 
Rev. Bras. Saúde Prod. Anim., Salvador, v.17, n.3, p.474-483 jul./set., $2016 \quad$ http://www.rbspa.ufba.br ISSN 15199940

valores de ganho de peso inferiores aos encontrados neste estudo, utilizando quatro diferentes suplementos injetáveis à base de aminoácidos em novilhos Nelore mantidos em pastagem de Brachiaria decumbens. Provavelmente, as diferenças no ganho de peso diário dos animais deste estudo em relação aos estudos dos autores citados acima estão relacionadas ao tipo de aminoácidos utilizados e a metodologia de aplicação empregada, uma vez que, diferem entre fabricantes.

Tabela 5. Parâmetros do modelo e medidas de qualidade do ajuste

\begin{tabular}{|c|c|c|c|c|}
\hline Função Logística & $y=\beta_{1} /\left(1+\exp \left(\left(\beta_{2}-x\right) / \beta_{3}\right)\right)+$ & & $\mathrm{R}^{2}$ & RMSE \\
\hline Test & $\mathrm{y}=62,664 /(1+\exp ((50,748-x) / 24,00$ & & & \\
\hline Parâmetros & $\begin{array}{rr}\beta_{1} & \beta_{2} \\
62,664^{* * *} & 50,748^{* * *}\end{array}$ & $\begin{array}{c}\beta_{3} \\
24,004^{* * *}\end{array}$ & 0,8971 & 7,536 \\
\hline ProbL & $\mathrm{y}=57,212 /(1+\exp ((44,225-\mathrm{x}) / 19,09$ & & & \\
\hline Parâmetros & $\begin{array}{cc}\beta_{1} & \beta_{2} \\
57,212^{* * *} & 44,225^{* * *}\end{array}$ & $\begin{array}{c}\beta_{3} \\
19,098^{* * *}\end{array}$ & 0,9303 & 6,152 \\
\hline ProbL+AAsPo & $\mathrm{y}=60,256 /(1+\exp ((41,580-\mathrm{x}) / 19,43$ & & & \\
\hline Parâmetros & $\begin{array}{cc}\beta_{1} & \beta_{2} \\
60,256^{* * *} & 41,580^{* * *}\end{array}$ & $\begin{array}{c}\beta_{3} \\
19,435^{* * *}\end{array}$ & 0,9444 & 5,737 \\
\hline ProbL+AAsInj & $\mathrm{y}=59,750 /(1+\exp ((51,866-\mathrm{x}) / 19,63$ & & & \\
\hline Parâmetros & $\begin{array}{cc}\beta_{1} & \beta_{2} \\
59,750^{* * *} & 51,866^{* * *}\end{array}$ & $\begin{aligned} \beta_{3} \\
19,637^{* * *}\end{aligned}$ & 0,9399 & 5,844 \\
\hline
\end{tabular}

Test - Testemunha; ProbL - Probiótico líquido; ProbL+AasPo - Probiótico líquido mais aminoácidos em pó; e ProbL+AasInj - Probiótico líquido mais aminoácidos injetável; $\gamma=$ ganho de peso; $x=$ tempo (dias); $\beta_{1}=$ assíntota, quando $x \rightarrow+\infty$, ou seja, o máximo valor teórico de $y(x) ; \beta_{2}=$ tempo de "meiavida", ou seja, o valor de $x$ correspondente $\beta_{1} / 2 ; \beta_{3}=$ taxa de crescimento médio de $y(x)$. *** Significativo a 0,001 de probabilidade.
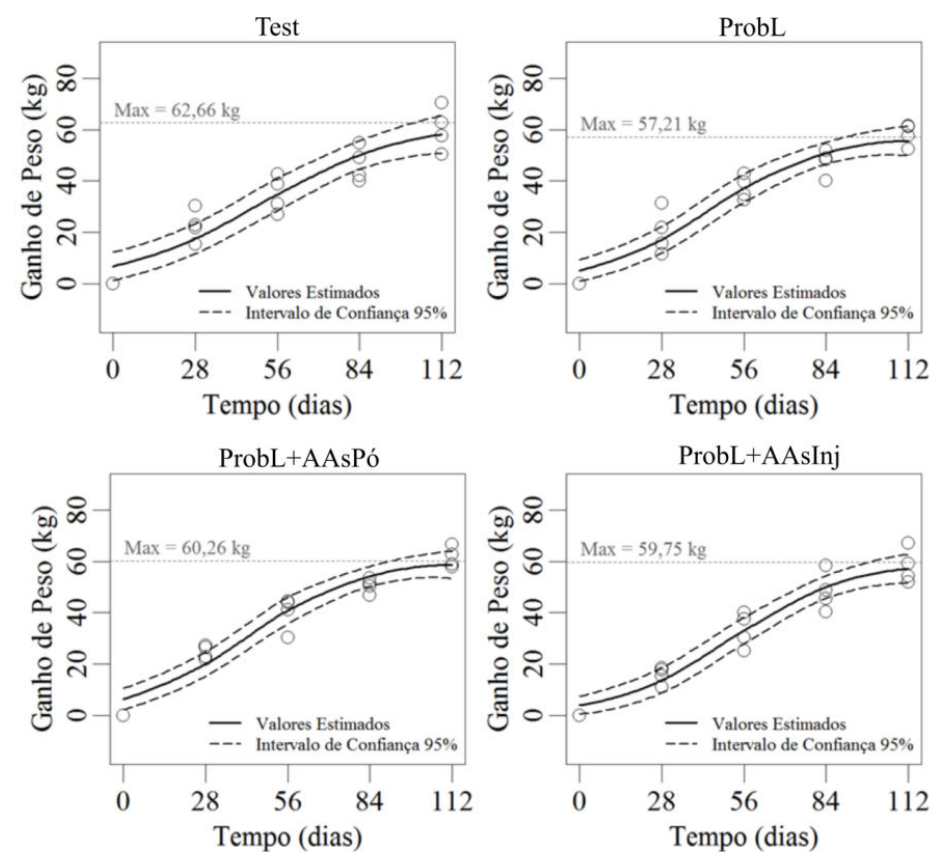

Figura 1. Ajuste do modelo de ganho de peso de novilhos suplementados com probióticos e/ou aminoácidos em função do tempo 
Rev. Bras. Saúde Prod. Anim., Salvador, v.17, n.3, p.474-483 jul./set., $2016 \quad \underline{\text { http://www.rbspa.ufba.br }}$ ISSN 15199940

Tabela 6. Médias e desvio padrão do peso corpóreo e do ganho de peso em novilhos suplementados com probióticos e/ou aminoácidos

\begin{tabular}{|c|c|c|c|c|}
\hline \multirow{2}{*}{ Tratamentos } & \multicolumn{2}{|c|}{ Peso Corpóreo (kg) } & \multicolumn{2}{|c|}{ Ganho de Peso (kg) } \\
\hline & Inicial & Final & Diário & Total \\
\hline Test & $396,31 \pm 30,04$ & $456,62 \pm 35,21$ & $0,538 \pm 0,12$ & $60,31 \pm 13,27$ \\
\hline ProbL & $390,44 \pm 28,31$ & $448,69 \pm 27,71$ & $0,520 \pm 0,10$ & $58,25 \pm 10,99$ \\
\hline ProbL+AAsPó & $399,62 \pm 32,14$ & $461,25 \pm 31,54$ & $0,550 \pm 0,09$ & $61,62 \pm 9,98$ \\
\hline ProbL+AAsInj & $395,25 \pm 26,99$ & $453,44 \pm 31,69$ & $0,519 \pm 0,09$ & $58,19 \pm 9,99$ \\
\hline
\end{tabular}

Test $=$ Testemunha; ProbL $=$ Probiótico líquido; ProbL + AasPo $=$ Probiótico líquido mais aminoácidos em pó; e ProbL+AasInj - Probiótico líquido mais aminoácidos injetável.

O teste da razão de verossimilhança é um teste de hipóteses que compara a qualidade do ajuste de dois modelos, desde que um dos modelos seja um caso especial do outro. Esse teste é utilizado como base para aplicar o teste de identidade de modelos, em que é possível averiguar se dois modelos são estatisticamente iguais, baseando-se no fato de que um modelo pode ser utilizado para representar um fenômeno real; fenômenos (tratamentos) podem ser considerados iguais desde que seus respectivos modelos apresentem identidade. Para modelos de regressão não lineares, com dados de repetições de um delineamento inteiramente casualizado, como foi o caso deste estudo, o teste de razão de verossimilhança, pode ser aplicado com aproximação pela estatística $\mathrm{F}$, (REGAZZI \& SILVA, 2010) principalmente por controlar mais o erro tipo I, independentemente do número de dados experimentais (N). Esse teste também pode ser utilizado para comparar tratamentos em situações em que os testes de comparações de médias (exemplo: teste Tukey) não são indicados, como em experimentos com fatores de origem quantitativa (exemplo: tempo). De acordo com o teste de razão de verossimilhança, observa-se que não houve diferença significativa entre os tratamentos (Tabela 7). Segundo Vanbelle et al. (1990), Corrêa et al. (1998), Campos Neto et al. (2004) e Morais et al. (2011), probióticos e aminoácidos promovem alteração no metabolismo animal, refletindo principalmente em um maior ganho de peso. No entanto, a utilização de ProbL, AAsPó e AAsInj, não apresentaram diferenças no aumento do ganho de peso de novilhos castrados, da raça Canchim e cruzados Canchim x Nelore.

Tabela 7. Teste de razão de verossimilhança entre os tratamentos

\begin{tabular}{lccc}
\hline Comparação & Valor F & p-valor & Decisão \\
\hline Test vs ProbL & 0,1821 & 0,9078 & Igual \\
Test vs ProbL+AAsPó & 0,7883 & 0,5088 & Igual \\
Test vs ProbL+AAsInj & 0,4586 & 0,7129 & Igual \\
ProbL vs ProbL+AAsPó & 0,8203 & 0,4916 & Igual \\
ProbL vs ProbL+AAsInj & 0,5853 & 0,6287 & Igual \\
ProbL+AAsPó vs ProbL+AAsInj & 2,4563 & 0,0797 & Igual \\
\hline
\end{tabular}

Test $=$ Testemunha; ProbL $=$ Probiótico líquido; ProbL + AasPo $=$ Probiótico líquido mais aminoácidos em pó; e ProbL+AasInj = Probiótico líquido mais aminoácidos injetável. 
Os resultados encontrados neste estudo corroboram com os encontrados por Figueiredo et al. (2012) que avaliando a suplementação com resíduo úmido de cervejaria, associado a um modificador orgânico à base de aminoácidos, vitaminas e minerais na terminação de novilhos Nelore em pastagem de Brachiaria brizantha cv. Marandú, não observaram aumento no ganho de peso dos animais. Do mesmo modo que Schuz Neto et al. (2013), ao trabalharem com novilhos Nelore em pastagem de Panicum maximum suplementados com probiótico adicionado ao sal mineral. No entanto, Rasteiro et al. (2007) encontraram resultados contrários, com aumento no ganho de peso de novilhos da raça Nelore em pastagem de Brachiaria brizantha suplementados com mistura mineral proteinada mais probiótico, assim como Campos Neto et al. (2003) trabalhando com fêmeas Nelore em pastagem de Brachiaria decumbens reportaram resultados superiores dos animais suplementados com aminoácido injetável de longa ação em relação aos animais testemunha.

O desempenho dos bovinos está relacionado com a proporção de energia e proteína da dieta, que estimula o crescimento de microrganismos ruminais, melhorando o aproveitamento dos nutrientes da pastagem, e com isso aumentando a produção de ácidos graxos voláteis (AGV) que são utilizados como fonte de energia pelos ruminantes. Nesse sentido, era esperado que o uso de probióticos e compostos à base de aminoácidos aumentassem o ganho de peso dos animais, principalmente no período da seca, quando as pastagens estão deficitárias em proteína, energia, mineral e vitaminas, e apresentam um nível elevado de fibra. Neste trabalho, o ganho de peso não significativo entre os tratamentos avaliados, pode estar relacionado à baixa relação entre energia e proteína da dieta oferecida aos animais. Com isso pode ter ocorrido uma menor eficiência metabólica em relação ao uso de probióticos e aminoácidos utilizados neste estudo, uma vez que, segundo Rasteiro et al. (2007) e Campos Neto et al. (2004) a relação de energia e proteína da dieta deve ser preconizada, pois existe uma interação positiva entre probióticos e aminoácidos com a suplementação proteica-energética da dieta. Outra possibilidade seria de não ter ocorrido limitação nutricional aos animais e isto ter levado a nenhuma diferença entre os tratamentos utilizados.

De acordo com o teste de identidade de modelos, conclui-se que não houve diferença significativa entre os modelos para os tratamentos utilizados, não havendo diferença no ganho de peso de novilhos, mantidos em pastagens de Brachiaria brizantha, com a utilização de probióticos e/ou de aminoácidos. O teste de identidade de modelos pode ser utilizado para avaliar o uso de aditivos na dieta de bovinos de corte.

\section{AGRADECIMENTOS}

Á CAPES, CNPq, FUNDECT, MCT, MEC, Governo Federal, Centro de Pesquisa do Pantanal (CPP) e Rede Pró-Centro-Oeste pelo financiamento deste trabalho.

\section{REFERÊNCIAS}

BALDWIN, R.L.; CALVERT, C.C.; HANIGAN, M.D.; BECKETT, J. Modelling Amino Acid Metabolism in Ruminants. In: D’MELLO, J.P.F. Amino acids in farm animal nutrition. Edinburg, UK: Cab International,1994. p.281-306. 
Rev. Bras. Saúde Prod. Anim., Salvador, v.17, n.3, p.474-483 jul./set., $2016 \quad \underline{\text { http://www.rbspa.ufba.br }}$ ISSN 15199940

CAMPOS NETO, O.; SCALZO, A.L.; CORREAA, I.; PARDO, F.J.D.

Avaliação do suplemento de aminoácidos injetável (aminofort), no desenvolvimento de novilhas da raça nelore. Revista Científica Eletrônica de Medicina Veterinária, n. 1, 2003.

CAMPOS NETO, O.; SCALZO, A.L.; MARCOS JUNIOR, G.; PARDO, F.J.D.; PIAZENTI, K.E.; SILVA, R.C. Interação do suplemento de aminoácidos injetável com sal mineral e sal mineral protéico-energético no desenvolvimento de bovinos. Revista Científica Eletrônica de Medicina Veterinária, n.2, 2004.

CARNEIRO, A.P.S.; MUNIZ, J.A.; CARNEIRO, P.L.S.; MALHADO, C.H.M.M.; MARTINS FILHO, R.; SILVA, F.F. Identidade de modelos não lineares para comparar curvas de crescimento de bovinos da raça Tabapuã. Pesquisa Agropecuária Brasileira, v.49, n.1, p.57-62, 2014.

CASTRO, S.R.S.; GRACIA, A.R.; VIANA, R.B.; NAHÚM, B.S.; COSTA, N.A.; ARAÚJO, V.; BENIGNO, R.N.M. Uso de antihelmínticos e bioestimulantes no desempenho de bovinos de corte suplementados a pasto no estado do Pará. Ciência Animal Brasileira, v.10, n.2, p.527-537, 2009.

CORRÊA, M.G.P.; CORRÊA, I.; LEMOS, A.M.; MARIN, J.M.;

NORTE, A.L. Influência da suplementação com aminoácidos sobre o ganho de peso em bovinos no período da entressafra. Revista do Instituto Laticínios Cândido Tostes, v.53, n.305, p.53-55, 1998.
EMBRAPA. Capim-massai (Panicum maximum cv. Massai) alternativa para diversificação de pastagem. Embrapa Gado de Corte. Campo Grande, 2001. 9p. (Comunicado técnico, 69). Disponível em: $<$ http://www.cnpgc.embrapa.br/publicac oes/cot/ COT69.html $>$. Acesso em: 15 maio 2016.

FIGUEIREDO, H.F.; FATURI, C.; RODRIGUES, L.F.S.; MANGAS, T.P.; RAMOS, A.F.O.; CARDOS, A.M. Terminação de bovinos de corte em pasto com suplementação de resíduo úmido de cervejaria associado ao uso de modificadores orgânicos e ivermectina. Revista de Ciências Agrárias, v.55, n.1, p.26-53, 2012.

MORAIS, J.A. da; BERCHIELLI, T.T.; REIS, R.A. Aditivos. In: BERCHIELLI, T. T.; PIRES, A V.; OLIVEIRA, S. G. de. Nutrição de Ruminantes. 2.ed. Jaboticabal: FUNEP, 2011. p.565- 599.

RASTEIRO, V.S.; BREMER-NETO, H.; ARENAS, S.E.; REIS, L.S.L.S.; FRAZATTI-GALLINA, N.M.; OBA, E.; PARDO P.E. Adição de probiótico na mistura mineral eleva o ganho de peso de bovinos no período da seca. Archivos Latino americano de Producción Animal, v.15, n.3, p. 8387, 2007.

RATKOWSKY, D.A. Handbook of nonlinear regression models. New York: Marcel Dekker, 1990. 241p.

REGAZZI, A.J; SILVA, C.H.O. Testes para verificar a igualdade de parâmetros e a identidade de modelos de regressão não-linear em dados de experimento com delineamento em blocos casualizados. Revista Ceres, v.57, p.315-320, 2010. 
Rev. Bras. Saúde Prod. Anim., Salvador, v.17, n.3, p.474-483 jul./set., $2016 \quad \underline{\text { http://www.rbspa.ufba.br }}$

SCHUZ NETO, C.; PACHECO, A.M.;

MONTANHA, F.P.; PARDO, P.E.;

PENHA, L.C.; BREMER NETO, H.

Efeitos da suplementação com

probióticos em relação a suplementação

com mistura mineral no ganho de peso de

garrotes nelore a pastejo intensivo de

Panicum maximum. Revista Científica

Eletrônica de Medicina Veterinária, $n$. 20, 2013.

SILVA, F. de L.; ALENCAR, M.M. de; FREITAS, A.R. de; PACKER, I.U.; MOURÃO, G.B. Curvas de crescimento em vacas de corte de diferentes tipos biológicos. Pesquisa Agropecuária

Brasileira, v.46, p.262-271, 2011.

SILVA, F.F.; VALADARES FILHO, S.C.; ITAVO, L.V.; VELOSO, C.M.; VALADARES, F.R.D.; CECON, P.R.; MORAES, E.H.B.K.; PAULINO, P.V.R. Exigências liquidas de aminoácidos para ganho de peso de nelore não castrado. Revista Brasileira de

Zootecnia, v.31, n.2, p.765-775, 2002.

SOUTELLO, R.V.G.; SILVA, C.L.S.P.; LIMA, M.A.; BAIER, M.O. Teste comparativo de ganho de peso em novilhos utilizando diferentes tipos de suplementos vitamínicos injetáveis. Revista de Ciências Agrárias e da Saúde, v.2, n.1, p.18-20, 2002.

SOUZA, L. de A.; CAIRES, D.N.; CARNEIRO, P.L.S.; MALHADO, C.H.M.; MARTINS FILHO, R. Curvas de crescimento em bovinos da raça Indubrasil criados no Estado de Sergipe.

Revista Ciência Agronômica, v.41, p.671-676, 2010.

VANBELLE, M.; TELLER, E.; FOCANT, M. Probiotics in animal nutrition: areview. Archives of Animal Nutrition, v.40, n.7, p.543-567, 1990.

Data de recebimento: 16/10/2015

Data de aprovação: 20/07/2016 